\title{
The Evolution of Discontinuities in Solutions of Homogeneous Nonlinear Hyperbolic Equations Having Smooth Initial Data
}

\author{
ALAN JEFFREY
}

Communicated by I. N. SNEDDoN

Introduction. The formation of discontinuities in solutions of nonlinear hyperbolic equations subject to Lipschitz continuous initial data has long been of interest. (See, for example, [1] to [7].) In the wave motion associated with problems of this type there is a clearly defined wavefront, a characteristic, along which the initial Lipschitz discontinuity propagates until, in certain cases, it tends to a jump discontinuity at some critical time. At this time the derivative of the solution becomes unbounded. The condition for the nonoccurrence of such a jump discontinuity, known as an exceptional case, and the equations determining the exact time of formation of a jump discontinuity on the wavefront in the regular, or genuinely nonlinear, case have been discussed in detail elsewhere for hyperbolic systems of first and higher order. ([4], [5], [6] and [7].)

However, so far, little attention has been paid to the problem of the evolution of discontinuities from smooth initial data. Possibly the first conjecture concerning a simplified problem of this type was that due to Riemann [8] who considered the conditions for a simple wave to develop a discontinuity. More recently Ludford [9] has re-examined this conjecture in the context of the general initial value problem for unsteady isentropic perfect gas flow by using a variant of the hodograph method. (See, for example, [1], Sections 21 and 30.) Unlike Stocker and Meyer [10] who examined the occurrence of limit lines to determine multiply-mapped flow regions, Ludford developed an unfolding procedure for initial curves in the hodograph plane, and then applied his method to obtain an asymptotic estimate of the time of breakdown of the solution.

In this paper, however, we shall not make use of either of these approaches. Instead, we shall consider a general homogeneous system of two first order 
quasi-linear hyperbolic equations and begin by deriving the equation governing the variation of a certain function of the $x$-derivative of a Riemann invariant along an appropriate characteristic. Then, after establishing that the solution of this equation is unique, we shall apply a useful comparison theorem which establishes the nature of the continuous dependence of this solution at any given time $t$ on the corresponding solution of a certain related comparison equation in terms of a parameter characterising the difference between the two equations. Then, by using two other simple comparison equations to respectively overestimate and underestimate the genuine solution, the finite times for which these comparison solutions exist are used to place bounds on the critical time $t_{c}$ at which the genuine solution develops a singularity along a characteristic. If it is assumed, as by Ludford, that the distribution of initial values along the initial line is such that the departure from constant values is always small, these bounds on $t_{c}$ become very close and may be used to provide a good estimate of the earliest time of breakdown of the solution of the original set of equations. The only requirement that need be placed on the initial values is that they be at least Lipschitz continuous. Thus the method permits the examination of the evolution of discontinuities from both Lipschitz continuous and smooth initial data.

Finally the results are applied to problems taken from the fields of gas dynamics, water waves and electrical transmission lines. The gas dynamic application confirms Ludford's simple result [9]; the water wave and transmission line applications are believed to be new, though when applied to a sinusoidal water wave disturbance propagating into a steady state they confirm the independently obtained results by Stoker [11] and Jeffrey [12] who assumed Lipschitz continuous initial data.

1. Basic equations and Riemann invariants. We shall be concerned with a homogeneous quasi-linear system of two first order partial differential equations of the form

$$
U_{t}+A U_{z}=0
$$

in which

$$
U=\left[\begin{array}{l}
u_{1} \\
u_{2}
\end{array}\right] \quad \text { and } \quad A=\left[\begin{array}{ll}
a_{11} & a_{12} \\
a_{21} & a_{22}
\end{array}\right],
$$

the suffixes $t$ and $x$ denoting partial differentiation with respect to $t$ and $x$.

It will be assumed further that the system of equations (1.1) is reducible, so that the coefficients of $A$ are functions only of the dependent variables $u_{1}$ and $u_{2}$, that these coefficients are continuous piecewise differentiable functions of $u_{1}$ and $u_{2}$, that the eigenvalues $\lambda^{(i)}(i=1,2)$ of the determinant

$$
|A-\lambda I|=0
$$

are real and distinct in the region of the $\left(u_{1}, u_{2}\right)$-dependent variable space 
in question, and that the corresponding left eigenvectors $l^{(i)}$ are linearly independent. The system (1.1) so considered is then said to be totally hyperbolic.

Pre-multiplying equation (1.1) by $l^{(i)}$ and using the relationship

$$
l^{(i)} A=\lambda^{(i)} l^{(i)}
$$

gives rise to the equations

$$
l^{(i)}\left(U_{t}+\lambda^{(i)} U_{x}\right)=0,
$$

for $i=1,2$, which are valid, respectively, along the $C^{(1)}$ and $C^{(2)}$ characteristic curves defined by

$$
C^{(i)}: \frac{d x}{d t}=\lambda^{(i)}, \quad \text { for } \quad i=1,2 .
$$

We now recognise [7, Section 2.1] that the expression $\left(U_{t}+\lambda^{(i)} U_{x}\right)$ involved in equation (1.4) simply denotes differentiation of $U$ with respect to time along the $i$-th characteristic. Let us now parameterise the $C^{(1)}$ characteristic curves by the requirement that the $C^{(1)}$ characteristic through the point $\left(0, t_{0}\right)$ in the $(x, t)$-plane has associated with it the constant value $\beta\left(t_{0}\right)$, where $\beta(t)$ is any monotonic differentiable function of $t$. Thus $\beta$ has a different constant value along each $C^{(1)}$ characteristic. The $C^{(2)}$ characteristics may be similarly parameterised in terms of a monotonic differentiable function $\alpha(t)$ so that $\alpha$ has a different constant value along each $C^{(2)}$ characteristic.

Since any parameterisation may be employed, subject only to the condition that $\alpha(t)$ and $\beta(t)$ be monotonic differentiable functions of $t$, we shall simply use the parameterisation $\alpha(t)=t, \beta(t)=t$, though for the time being, to avoid confusion in Section 2, we shall retain the symbols $\alpha$ and $\beta$ as new independent variables.

In terms of $\alpha$ and $\beta$, the two equations represented by (1.4) can then be written,

$$
l^{(1)} U_{\alpha}=0 \text { along } C^{(1)} \text { characteristics, }
$$

and

$$
l^{(2)} U_{\beta}=0 \text { along } C^{(2)} \text { characteristics. }
$$

Writing the row vector $l^{(i)}$ in the form $l^{(i)}=\left[l_{1}^{(i)}, l_{2}^{(i)}\right]$, equations (1.6) and (1.7) become

$$
l_{1}^{(1)} u_{1 \alpha}+l_{2}^{(1)} u_{2 \alpha}=0 \text { along } C^{(1)} \text { characteristics }
$$

and

$$
l_{1}^{(2)} u_{1 \beta}+l_{2}^{(2)} u_{2 \beta}=0 \text { along } C^{(2)} \text { characteristics. }
$$

Multiplying the respective equations by the integrating factors $q_{1}$ and $q_{2}$, if necessary, so that they become exact differentials and then integrating with respect to $\alpha$ and $\beta$, respectively, we finally arrive at the relations 


$$
\int q_{1} l_{1}^{(1)} d u_{1}+\int q_{1} l_{2}^{(1)} d u_{2}=r(\beta) \quad \text { along } C^{(1)} \text { characteristics }
$$

and

$$
\int q_{2} l_{1}^{(2)} d u_{1}+\int q_{2} l_{2}^{(2)} d u_{2}=s(\alpha) \quad \text { along } C^{(2)} \text { characteristics, }
$$

in which the integration constants $r(\beta)$ and $s(\alpha)$ are known as Riemann invariants. (These integrating factors might be necessary since the left eigenvector $l^{(i)}$, which is arbitrary up to a non-zero scalar factor, might not be in the appropriate form to make equations (1.8) and (1.9) exact differentials.)

As is usual in the discussion of reducible equations [1, Section 21], the relationship $\left(u_{1}, u_{2}\right) \rightarrow(r, s)$ is assumed to be one-to-one. The discontinuities that can occur in the resulting simple wave flows when this condition is not satisfied are simpler and have been discussed in detail elsewhere [1, 7].

It thus follows from equation (1.10) and the definition of differentiation along a characteristic direction that alternative forms of equations (1.8) and $(1.9)$ in terms of the Riemann invariants $r(\beta)$ and $s(\alpha)$ are

$$
\frac{d r}{d \alpha}=\frac{\partial r}{\partial t}+\lambda^{(1)} \frac{\partial r}{\partial x}=0 \quad \text { along } C^{(1)} \text { characteristics }
$$

and

$$
\frac{d s}{d \beta}=\frac{\partial s}{\partial t}+\lambda^{(2)} \frac{\partial s}{\partial x}=0 \quad \text { along } C^{(2)} \text { characteristics. }
$$

In the next Section we shall use these equations to derive important differential equations which are applicable along characteristics. The singularity in the solutions of these equations determines the time and position of occurrence of a singularity in the solution of equations (1.1) when suitable initial and boundary values are given.

2. Differential equations along characteristics. Since the expression $d r / d \alpha=0$ in equation (1.12) involves the behaviour of $r_{t}$ and $r_{x}$ along $C^{(1)}$ characteristics, we shall now use it to determine the differential equations satisfied by these functions along the $C^{(1)}$ characteristics. To achieve this we derive two new equations from equation (1.12) by differentiating it with respect to $\alpha$ and to $\beta$ to obtain

(2.1) $\left(\frac{\partial}{\partial t}+\lambda^{(1)} \frac{\partial}{\partial x}\right)\left(\frac{\partial r}{\partial t}+\lambda^{(1)} \frac{\partial r}{\partial x}\right)=0$ and $\left(\frac{\partial}{\partial t}+\lambda^{(2)} \frac{\partial}{\partial x}\right)\left(\frac{\partial r}{\partial t}+\lambda^{(1)} \frac{\partial r}{\partial x}\right)=0$,

which are respectively valid along $C^{(1)}$ characteristics. Since the system (1.1) is assumed to be totally hyperbolic, so that $\lambda^{(1)} \neq \lambda^{(2)}$, it then follows immediately that 


$$
\frac{\partial}{\partial t}\left(\frac{\partial r}{\partial t}+\lambda^{(1)} \frac{\partial r}{\partial x}\right)=0 \quad \text { and } \quad \frac{\partial}{\partial x}\left(\frac{\partial r}{\partial t}+\lambda^{(1)} \frac{\partial r}{\partial x}\right)=0
$$

along $C^{(1)}$ characteristics. $A s \lambda^{(1)}$ and $\lambda^{(2)}$ are finite, and $\partial r / \partial t$ and $\partial r / \partial x$ are related by equation (1.12), it will be sufficient to determine the time of breakdown of the solution due to the Riemann invariant $r$ by determining the time at which $\partial r / \partial x$ becomes unbounded.

We are thus led to consider the equation

$$
\frac{\partial}{\partial x}\left(\frac{\partial r}{\partial t}+\lambda^{(1)} \frac{\partial r}{\partial x}\right)=0 \quad \text { along } C^{(1)} \text { characteristics. }
$$

Performing the indicated differentiation then shows that

$$
\frac{\partial^{2} r}{\partial t \partial x}+\frac{\partial \lambda^{(1)}}{\partial r}\left(\frac{\partial r}{\partial x}\right)^{2}+\frac{\partial \lambda^{(1)}}{\partial s}\left(\frac{\partial s}{\partial x}\right)\left(\frac{\partial r}{\partial x}\right)+\lambda^{(1)} \frac{\partial^{2} r}{\partial x^{2}}=0
$$

must hold along $C^{(1)}$ characteristics. A similar result holds along $C^{(2)}$ characteristics for which strictly analogous reasoning shows that the equation

$$
\frac{\partial^{2} s}{\partial t \partial x}+\frac{\partial \lambda^{(2)}}{\partial s}\left(\frac{\partial s}{\partial x}\right)^{2}+\frac{\partial \lambda^{(2)}}{\partial r}\left(\frac{\partial r}{\partial x}\right)\left(\frac{\partial s}{\partial x}\right)+\lambda^{(2)} \frac{\partial^{2} s}{\partial x^{2}}=0
$$

describes the variation of $\partial s / \partial x$. This equation then determines the time of breakdown of the solution due to the Riemann invariant $s$.

Returning to equation (2.4) let us simplify it by first recognising that the first and last terms combine to form $d / d \alpha(\partial r / \partial x)$. Then, applying the operator identity $d / d \alpha \equiv d / d \beta+\left(\lambda^{(1)}-\lambda^{(2)}\right) \partial / \partial x$ to $s(\alpha)$, we see that $d s / d \alpha=$ $\left(\lambda^{(1)}-\lambda^{(2)}\right)(\partial s / \partial x)$ so that equation (2.4) reduces to

$$
\frac{d}{d \alpha}\left(\frac{\partial r}{\partial x}\right)+\left(\frac{\partial \lambda^{(1)}}{\partial r}\right)\left(\frac{\partial r}{\partial x}\right)^{2}+\frac{1}{\left(\lambda^{(1)}-\lambda^{(2)}\right)}\left(\frac{d s}{d \alpha}\right)\left(\frac{\partial \lambda^{(1)}}{\partial s}\right)\left(\frac{\partial r}{\partial x}\right)=0 .
$$

This homogeneous nonlinear ordinary differential equation along the $C^{(1)}$ characteristics can now be further simplified if we change the dependent variable so that only a quadratic term in the new dependent variable remains. To achieve this we now write $v_{1}=(\partial r / \partial x) f_{1}$, thereby causing equation (2.6) to take the form

$$
\frac{d v_{1}}{d \alpha}+\frac{1}{f_{1}}\left(\frac{\partial \lambda^{(1)}}{\partial r}\right) v_{1}^{2}=0,
$$

provided the function $f_{1}$ is chosen such that it satisfies the equation

$$
\frac{1}{f_{1}} \frac{d f_{1}}{d s}=\left(\frac{1}{\lambda^{(1)}-\lambda^{(2)}}\right)\left(\frac{\partial \lambda^{(1)}}{\partial s}\right) .
$$

Thus $f_{1}$ is given by the expression

$$
f_{1}=K \exp \left\{\int\left(\frac{1}{\lambda^{(1)}-\lambda^{(2)}}\right)\left(\frac{\partial \lambda^{(1)}}{\partial s}\right) d s\right\}
$$


where $K$ is an arbitrary constant which, for convenience, we shall assume to be unity. So, finally, if we define $g_{1}$ by the relation

$$
g_{1}=\int\left(\frac{1}{\lambda^{(1)}-\lambda^{(2)}}\right)\left(\frac{\partial \lambda^{(1)}}{\partial s}\right) d s,
$$

equation (2.7) determining the variation of the new dependent variable $v_{1}$ along the $C^{(1)}$ characteristics becomes

$$
\frac{d v_{1}}{d \alpha}+e^{-g_{1}}\left(\frac{\partial \lambda^{(1)}}{\partial r}\right) v_{1}^{2}=0 .
$$

Similar reasoning shows that if $v_{2}=(\partial s / \partial x) f_{2}$, where

$$
f_{2}=\exp \left\{\int\left(\frac{1}{\lambda^{(2)}-\lambda^{(1)}}\right)\left(\frac{\partial \lambda^{(2)}}{\partial r}\right) d r\right\}
$$

and $g_{2}$ is defined in a manner analogous to $g_{1}$, then

$$
\frac{d v_{2}}{d \beta}+e^{-o s}\left(\frac{\partial \lambda^{(2)}}{\partial s}\right) v_{2}^{2}=0
$$

along $C^{(2)}$ characteristics. Equations (2.11) and (2.13) will be taken as the starting point for the derivation of asymptotic estimates of the critical time $t_{c}$ at which the solution of the system of equations (1.1) first develops a singularity due to $v_{1}$ or $v_{2}$ becoming unbounded along their respective characteristics.

3. Uniqueness and comparison theorems. To pose an initial value problem for the system of equations (1.1) we must first specify the behaviour of the column vector $U$ on the initial line $t=0$. Let us set

$$
U(x, 0)=\Phi(x),
$$

where $\Phi(x)$ is some column vector whose elements depend only on $x$, and suppose that the elements of $\Phi(x)$ are at least Lipschitz continuous on the $x$-axis. Then, by virtue of equations (1.10) and (1.11), the vector $\Phi(x)$ determines the values of $r$ and $s$ which are to be associated with the $C^{(1)}$ and $C^{(2)}$ characteristics passing through each point of the $x$-axis. As the coefficients of the matrix $A(U)$ in equation (1.1) are assumed to be at least continuous and piecewise differentiable functions of $u_{1}$ and $u_{2}$, this also implies that the coefficients of $v_{1}^{2}$ and $v_{2}^{2}$ in equations (2.11) and (2.13) must be at least Lipschitz continuous.

Now equations (2.11) and (2.13) are both of the form

$$
\frac{d w}{d x}=A(x, w),
$$

in which $A(x, w)$ is Lipschitz continuous with respect to $w$ in an appropriate region of the $(x, w)$-plane. Thus, since by a well known result [13, Chapter 1 , Theorem 2.2] the solution $w$ of equation (3.2) is unique, it immediately follows that the solutions $v_{1}$ and $v_{2}$ of equations (2.11) and (2.13) are unique. 
As the nonlinear variable coefficient equations (2.11) and (2.13) determining the behaviour of $v_{1}$ and $v_{2}$ along the $C^{(1)}$ and $C^{(2)}$ characteristics cannot be integrated in the form shown we shall instead apply a useful comparison theorem. To see the nature of the problem that is involved let us begin by considering the equation (2.11) that is appropriate to a $C^{(1)}$ characteristic through the point $x=\xi$ on the initial line $t=0$. The coefficient $e^{-\sigma_{1}}\left(\partial \lambda^{(1)} / \partial r\right)$ in this equation is a function of $\alpha$ and $v_{1}(\alpha)$ when both the point $x=\xi$ on the initial line through which the defining $C^{(1)}$ characteristic passes and the initial vector $\Phi(x)$ have been specified.

We shall denote this coefficient simply by $-A(\alpha, \xi)$, so that the constant value $-A_{0}(\xi)$ assumed by it when $\alpha=\alpha_{0}$, say, at the point $x=\xi$ on the initial line will be determined by the relation $A_{0}(\xi)=A\left(\alpha_{0}, \xi\right)$. Let us now compare the solutions $v_{1}$ and $\bar{v}_{1}$ of the two equations

$$
\frac{d v_{1}}{d \alpha}=A(\alpha, \xi) v_{1}^{2}
$$

and

$$
\frac{d \bar{v}_{1}}{d \alpha}=A_{0}(\xi) \bar{v}_{1}^{2},
$$

respectively, where $v_{1}$ and $\bar{v}_{1}$ are subject to some initial conditions and $A(\alpha, \xi)$ is Lipschitz continuous with respect to $\alpha$. The comparison solution $\bar{v}_{1}$ is then the solution of a constant coefficient equation.

To simplify the argument we now introduce the Lipschitz continuous function $B\left(\alpha, v_{1}(\alpha), \xi\right)=A(\alpha, \xi) v_{1}^{2}$ with the Lipschitz constant $K(\xi)$, and the continuous function $B_{0}\left(\bar{v}_{1}(\alpha), \xi\right)=A_{0}(\xi) \bar{v}_{1}^{2}$. We further assume that they satisfy the condition

$$
\left|B(\alpha, w, \xi)-B_{0}(w, \xi)\right| \leqq M(\xi),
$$

for some range of $\alpha$, say, $\alpha_{0} \leqq \alpha \leqq \alpha_{1}$, where $\alpha_{1}$ is chosen such that $w$ remains uniformly bounded for all values of $A(\alpha, \xi)$ and $A_{0}(\xi)$ appropriate to all points $\xi$ on the initial line. That it is possible to choose such a value of $\alpha_{1}$ will be established later.

The final forms of the equations to be examined are thus

$$
\frac{d v_{1}}{d \alpha}=B\left(\alpha, v_{1}(\alpha), \xi\right)
$$

and

$$
\frac{d \bar{v}_{1}}{d \alpha}=B_{0}\left(\bar{v}_{1}(\alpha), \xi\right) .
$$

Hence, applying an obvious extension of a standard comparison theorem [13, Chapter 1, Theorem 2.1] it immediately follows that 


$$
\begin{aligned}
\left|v_{1}(\alpha)-\bar{v}_{1}(\alpha)\right| \leqq\left|v_{1}\left(\alpha_{0}\right)-\bar{v}_{1}\left(\alpha_{0}\right)\right| & \exp \left\{K(\xi)\left|\alpha-\alpha_{0}\right|\right\} \\
+ & \frac{M(\xi)}{K(\xi)}\left[\exp \left\{K(\xi)\left|\alpha-\alpha_{0}\right|\right\}-1\right] .
\end{aligned}
$$

Since it will be convenient to adopt the same initial conditions for the comparison solution $\bar{v}_{1}$ as for the genuine solution $v_{1}$, we may set $v_{1}\left(\alpha_{0}\right)=\bar{v}_{1}\left(\alpha_{0}\right)$ at all points of the initial line to obtain

$$
\left|v_{1}(\alpha)-\bar{v}_{1}(\alpha)\right| \leqq \frac{M(\xi)}{K(\xi)}\left[\exp \left\{K(\xi)\left|\alpha-\alpha_{0}\right|\right\}-1\right] .
$$

This provides a bound for the modulus of the error between the required solution $v_{1}$ and the simple comparison solution $\bar{v}_{1}$ at a point with coordinate $\alpha$ on the determining $C^{(1)}$ characteristic through $x=\xi$ on the initial line. A similar result is also valid for the solution $v_{2}$ of equation (2.13) and its related comparison solution $\bar{v}_{2}$.

Later we shall integrate the comparison equation (3.4) so that when the form of $e^{-\sigma_{1}}\left(\partial \lambda^{(1)} / \partial r\right)$ is specified and $K(\xi)$ and $M(\xi)$ are known, inequality (3.9) may be used to estimate the modulus of its difference from $v_{1}$ for $\alpha_{0} \leqq \alpha \leqq \alpha_{1}$. The discussion of the estimate for $v_{2}$ is strictly analogous to that for $v_{1}$ and so it now remains to show the existence of the number $\alpha_{1}$ introduced in connection with condition (3.5). We shall establish this in the following section prior to determining the asymptotic estimate of the critical time.

4. Asymptotic estimate of the critical time $t_{c}$. To determine the behavior of the comparison solution $\bar{v}_{1}$, say, as a function of the time $t$, we need only recall that the parameterisation $\alpha(t) \equiv t$ has been used along the $C^{(1)}$ characteristics so that direct integration of equation (3.4) immediately shows that

$$
\bar{v}_{1}(t)=\frac{\bar{v}_{01}(\xi)}{1-t A_{0}(\xi) \bar{v}_{01}(\xi)},
$$

where $\bar{v}_{01}(\xi)$ denotes the initial value of $\bar{v}_{1}$ at the point $x=\xi$ on the initial line $t=0$ through which the defining characteristic passes.

This expression shows that when $A_{0}(\xi) \bar{v}_{01}(\xi)$ is positive, the comparison solution $\bar{v}_{1}$ becomes unbounded on the defining $C^{(1)}$ characteristics at elapsed critical times $T_{c}^{(1)}\left(A_{0}(\xi), \xi\right)$ determined by the expression

$$
T_{c}^{(1)}\left(A_{0}(\xi), \xi\right)=\frac{1}{A_{0}(\xi) \bar{v}_{01}(\xi)} .
$$

The superscript 1 and the argument $\xi$ are used to signify that $T_{c}^{(1)}\left(A_{0}(\xi), \xi\right)$ is the comparison solution critical time on the $C^{(1)}$ characteristic that passes through the point with coordinate $x=\xi$ on the initial line $t=0$.

Similar reasoning then shows that the comparison solution $\bar{v}_{2}$ becomes unbounded on the defining $C^{(2)}$ characteristics at a critical time determined by the expression 


$$
T_{c}^{(2)}\left(\bar{A}_{0}(\eta), \eta\right)=\frac{1}{\bar{A}_{0}(\eta) \bar{v}_{02}(\eta)} .
$$

The superscript 2 signifies that $T_{c}^{(2)}\left(\bar{A}_{0}(\eta), \eta\right)$ is the comparison solution critical time on the $C^{(2)}$ characteristic passing through the point with coordinate $x=\eta$ on the initial line $t=0$. In this equation $\bar{A}_{0}(\eta)$ has been used to denote the constant coefficient in the comparison equation for $\bar{v}_{2}$ that corresponds to the coefficient $A_{0}(\xi)$ in equation (3.4).

Provided the derivatives $\left(\partial \lambda^{(1)} / \partial r\right)$ and $\left(\partial \lambda^{(2)} / \partial s\right)$ do not change sign along the initial line, by always choosing the signs of the Riemann invariants so that these derivatives are negative, the coefficients $A_{0}(\xi)$ and $\bar{A}_{0}(\eta)$ may always be taken as positive, thereby ensuring that the elapsed critical times given by (4.2) and (4.3) are positive whenever $\bar{v}_{01}, \bar{v}_{02}$ are positive.

Let us now establish the existence of the number $\alpha_{1}$ used in condition (3.5) and examine the relationship of the comparison solution critical time $T_{c}^{(1)}(A(\xi), \xi)$ to the critical time $t_{c}^{(1)}(\xi)$ that is appropriate to $v_{1}$. Again the result for $v_{2}$ is strictly analogous and so only the details of the argument for the variable $v_{1}$ will be presented.

We begin by considering equation (3.4) defined along the $C^{(1)}$ characteristic through the point $x=\xi$ on the initial line (hereafter replacing $\alpha$ by $t$ ) together with the new comparison equation

$$
\frac{d \tilde{v}_{1}}{d t}=N \tilde{v}_{1}^{2}
$$

in which $N$ is some positive constant. We shall assume that $\tilde{v}_{1}=v_{1}$ when $t=0$, so that equations (3.3) and (4.4) have identical initial conditions.

If equation (3.3) is now subtracted from this equation it is easily established that we may write the result in the form

$$
\frac{d}{d t}\left[\tilde{v}_{1}-v_{1}\right]=[N-A(t, \xi)] \tilde{v}_{1}^{2}+A(t, \xi)\left[\tilde{v}_{1}^{2}-v_{1}^{2}\right] .
$$

Using the initial condition $v_{1}=\tilde{v}_{1}$ when $t=0$ then shows that the rate and direction of change of the difference $\left(\tilde{v}_{1}-v_{1}\right)$ is determined by the magnitude and sign of $N-A(t, \xi)$. When, for example, $A(t, \xi)>0$ and it is possible to choose a positive $N$ dependent on $\xi$, which we shall write as $N(\xi)$, such that $N(\xi)-A(t, \xi)>0$ throughout the range of integration with respect to time $t$, we may at once conclude that $v_{1}<\delta_{1}$ and so $\tilde{v}_{1}$ then provides an upper bound for $v_{1}$ on the defining characteristic. Since we have seen from equation (4.2) that for a comparison equation of this kind $\tilde{v}_{1}$ will cease to be defined beyond the time

$$
T_{c}^{(1)}(N(\xi), \xi)=\frac{1}{N(\xi) \tilde{v}_{01}(\xi)},
$$

it immediately follows that along the characteristic that is involved $T_{c}^{(1)}(N(\xi), \xi)$ provides a lower bound for the time for which the exact solution $v_{1}$ exists. 
Provided $A(t, \xi)$ does not change sign along a characteristic (i.e., $\partial \lambda^{(1)} / \partial r$ and $\partial \lambda^{(2)} / \partial s$ remain negative everywhere), by taking $N(\xi)$ to be the least upper bound $n(\xi)$ of $A(t, \xi)$ along the characteristic we obtain the expression $T_{\mathbf{i n f}}^{(1)}(\xi)=$ $1 / n(\xi) \tilde{v}_{01}(\xi)$ for the greatest lower bound of the times $T_{c}^{(1)}(N(\xi), \xi)$. This then is the best estimate that may be obtained by this method of the lower bound for the critical time for the genuine solution $v_{1}$ associated with the $C^{(1)}$ characteristic through $x=\xi$ on the initial line.

To obtain the analytic form of $T_{\text {inf }}^{(1)}(\xi)$ we must now utilize the results

$$
N(\xi)=-\left(\frac{\partial \lambda^{(1)}}{\partial r}\right) \exp \left\{-g_{1}(r, s)\right\}, \quad \sigma_{01}(\xi)=\left[\left(\frac{\partial r}{\partial x}\right) \exp \left\{g_{1}(r, s)\right\}\right]_{t=0} .
$$

It will be recalled that $x=\xi$ on the initial line determines a particular $C^{(1)}$ characteristic, and so corresponds to some definite value of $r$ which we shall denote by $r_{0}(\xi)$ whilst $s$, which varies along the $C^{(1)}$ characteristic, is some function of time with the initial value $s=s_{0}(\xi)$ at $t=0$, since on the initial line $r(x, t)$ and $s(x, t)$ are of the form $r_{0}(x)=r(x, 0)$ and $s_{0}(x)=s(x, 0)$. The expression for $T_{\mathbf{i n f}}^{(1)}(\xi)$ then becomes

$$
T_{\text {int }}^{(1)}(\xi)=\frac{-1}{\left(\frac{\partial r}{\partial x}\right)_{\substack{t=0 \\ z=\xi}} \exp \left\{g_{1}\left(r_{0}(\xi), s_{0}(\xi)\right)\right\} \sup \left[\left(\frac{\partial \lambda^{(1)}}{\partial r}\right) \exp \left\{-g_{1}\left(r_{0}(\xi), s\right)\right\}\right]},
$$

where sup $[\cdot]_{0}$ denotes the supremum with respect to $s$, the variable $r_{0}$ being held constant.

Each $C^{(1)}$ characteristic has such a time $T_{\text {inf }}^{(1)}(\xi)$ associated with it and if we denote the greatest lower bound of $T_{\mathrm{in}}^{(1)}(\xi)$ by ${ }_{*} T^{(1)}$, then ${ }_{*} T^{(1)}$ is the desired lower bound of the times for which the comparison solution exists.

The critical time ${ }_{*} T^{(1)}$ associated with the $C^{(1)}$ characteristics thus has the form

$$
{ }_{*} T^{(1)}=\inf \left[\frac{-1}{\left(\frac{\partial r}{\partial x}\right)_{\substack{t=0 \\ x=\xi}} \exp \left\{g_{1}\left(r_{0}(\xi), s_{0}(\xi)\right)\right\} \sup \left[\left(\frac{\partial \lambda^{(1)}}{\partial r}\right) \exp \left\{-g_{1}\left(r_{0}(\xi), s\right)\right\}\right]}\right] \text {, }
$$

and is the least upper bound of the numbers $\alpha_{1}$ involved in inequality (3.5) as $\xi$ ranges over the initial line. That this bound exists follows immediately from the fact that for a properly posed problem, $\Phi(x)$ and the initial Riemann invariant distributions $r_{0}(x)=r(x, 0)$ and $s_{0}(x)=s(x, 0)$ are continuous and finitely bounded functions so that $T_{\text {inf }}^{(1)}(\xi)$, which is a continuous function of them, must itself be finitely bounded. The time ${ }_{*} T^{(1)}$ must be positive, so that a breakdown in the solution due to the $C^{(1)}$ characteristics will occur, whenever

$$
\left(\frac{\partial r}{\partial x}\right)_{\substack{c=0 \\ x=\xi}}>0
$$


This establishes the existence of a number $\alpha_{1}<{ }_{*} T^{(1)}$ with the property that the function $w$ in inequality (3.5) remains uniformly bounded for all points $\xi$ on the initial line provided $\alpha_{0}<\alpha<\alpha_{1}$.

A similar argument when applied to the $C^{(2)}$ characteristics gives rise to a critical time ${ }_{*} T^{(2)}$ associated with the $C^{(2)}$ characteristics of the form

$$
\left.{ }_{(4.7)} T^{(2)}=\inf \left[\frac{-1}{\left(\frac{\partial s}{\partial x}\right)_{\substack{t=0 \\ x=\eta}} \exp \left\{g_{2}\left(r_{0}(\eta), s_{0}(\eta)\right)\right\} \sup \left[\left(\frac{\partial \lambda^{(1)}}{\partial s}\right) \exp \left\{-g_{2}\left(r, s_{0}(\eta)\right)\right\}\right.}\right]_{r}\right]_{\eta} \text {. }
$$

Since both the $C^{(1)}$ and $C^{(2)}$ characteristics can give rise to a breakdown of the solution, it finally follows that the number $t_{\text {inf }}$, defined to be the least positive number of ${ }_{*} T^{(1)}$ and ${ }_{*} T^{(2)}$, provides the lower bound for the time of existence of a solution of the comparison equations. Since $v_{i}$ majorizes $v_{i}$ it follows also that $t_{\mathrm{int}}$ is the best estimate obtainable by this method of the lower bound for the time of existence of a solution of the original system of equations (1.1).

Returning to equation (4.4) and this time identifying the number $N$ dependent on $\xi$ with the number $N^{\prime}(\xi)$ which now satisfies the inequality $N^{\prime}(\xi)-A(t, \xi)<$ 0 , the previous argument then establishes that $\sigma_{1}<v_{1}$. Thus, on the defining $C^{(1)}$ characteristic, the corresponding time $T_{c}^{(1)}\left(N^{\prime}(\xi), \xi\right)$ provides an upper bound to the time of existence of a solution for $\tilde{v}_{1}$ and thus also for $v_{1}$. Continuing the argument in an analogous fashion then leads to the determination of a number $t_{\text {sup }}$ which is the best estimate obtainable by this method of the upper bound for the time of existence of a solution of the original system of equations (1.1). The number $t_{\text {sup }}$ is the least positive number of the two expressions

$$
{ }^{*} T^{(1)}=\inf \left[\frac{-1}{\left(\frac{\partial r}{\partial x}\right)_{\substack{c=0 \\ x=\xi}} \exp \left\{g_{1}\left(r_{0}(\xi), s_{0}(\xi)\right)\right\} \inf \left[\left(\frac{\partial \lambda^{(1)}}{\partial r}\right) \exp \left\{-g_{1}\left(r_{0}(\xi), s\right)\right\}\right]}\right]_{\xi},
$$

and

$$
{ }^{*} T^{(2)}=\inf \left[\frac{-1}{\left(\frac{\partial s}{\partial x}\right)_{\substack{t=0 \\ x=1}} \exp \left\{g_{2}\left(r_{0}(\eta), s_{0}(\eta)\right)\right\} \inf \left[\left(\frac{\partial \lambda^{(2)}}{\partial s}\right) \exp \left\{-g_{2}\left(r, s_{0}(\eta)\right)\right\}\right]}\right] \text {. }
$$

The actual value $t_{\mathrm{c}}$ of the time of existence of a solution of the original system of equations (1.1) thus satisfies the inequality

$$
t_{\text {int }}<t_{c}<t_{\text {sup }} .
$$

The numbers $t_{\text {int }}$ and $t_{\text {sup }}$ are to be interpreted in the sense that the solution is certainly bounded for $t<t_{\text {int }}$, whilst the solution is certainly unbounded for $t>t_{\text {sup }}$. 
When, as assumed by Ludford [9], the Riemann invariant distributions $r_{0}(x)$ and $s_{0}(x)$ differ only slightly from the constant values $\hat{r}_{0}$ and $\hat{s}_{0}$, respectively, then $\left(t_{\text {sup }}-t_{\text {inf }}\right)$ is small and the bounds provide a good estimate of $t_{c}$. Under these conditions, since $r$ and $s$ are constant along respective characteristics, they also can differ only slightly from $\hat{r}_{0}$ and $\hat{s}_{0}$ at all subsequent times along the characteristics until breakdown of the solution, so that continuous functions of $r$ and $s$ will vary only slightly from constant values. Consequently, the expressions occurring in (4.6) to (4.9) which define $t_{\mathrm{inf}}$ and $t_{\mathrm{sup}}$ may then be approximated by the much simpler expressions:

$t_{\text {inf }}$ is the least positive number of the two quantities

$$
\frac{-1}{\max _{r, s}\left[\left(\frac{\partial \lambda^{(1)}}{\partial r}\right) \exp \left\{g_{1}\left(\hat{r}_{0}, \hat{s}_{0}\right)-g_{1}\left(\hat{r}_{0}, s\right)\right\}\right] \max \left(\frac{\partial r}{\partial x}\right)_{t=0}},
$$

$$
\frac{-1}{\max _{r, s}\left[\left(\frac{\partial \lambda^{(2)}}{\partial s}\right) \exp \left\{g_{2}\left(\hat{r}_{0}, \hat{s}_{0}\right)-g_{2}\left(r, \hat{s}_{0}\right)\right\}\right] \max \left(\frac{\partial s}{\partial x}\right)_{t=0}}
$$

and

$t_{\text {sup }}$ is the least positive number of the two quantities

$$
\begin{aligned}
& \frac{-1}{\min _{r, s}\left[\left(\frac{\partial \lambda^{(1)}}{\partial r}\right) \exp \left\{g_{1}\left(\hat{r}_{0}, \hat{s}_{0}\right)-g_{1}\left(\hat{r}_{0}, s\right)\right\}\right] \max \left(\frac{\partial r}{\partial x}\right)_{t=0}}, \\
& \frac{-1}{\min _{r, s}\left[\left(\frac{\partial \lambda^{(2)}}{\partial s}\right) \exp \left\{g_{2}\left(\hat{r}_{0}, \hat{s}_{0}\right)-g_{2}\left(r, \hat{s}_{0}\right)\right\}\right] \max \left(\frac{\partial s}{\partial x}\right)_{t=0}} \text {. }
\end{aligned}
$$

A further simplification is possible if the functions $g_{1}(r, s)$ and $g_{2}(r, s)$ are represented approximately by the first two terms of their Taylor series expansions and the defining relation (2.10) for $g_{1}(r, s)$ and the corresponding relation for $g_{2}(r, s)$ are used. To see this we write

$$
g_{1}\left(\hat{r}_{0}, s\right)=g_{1}\left(\hat{r}_{0}, \hat{s}_{0}\right)+\left(s-\hat{s}_{0}\right)\left(\frac{\partial g_{1}}{\partial s}\right)_{0}+O\left(\left(s-\hat{s}_{0}\right)^{2}\right)
$$

which, by virtue of equation (2.10), becomes

$$
g_{1}\left(\hat{r}_{0}, \hat{s}_{0}\right)-g_{1}\left(\hat{r}_{0}, s\right)=\left(\frac{\hat{s}_{0}-s}{\lambda_{0}^{(1)}-\lambda_{0}^{(2)}}\right)\left(\frac{\partial \lambda^{(1)}}{\partial s}\right)_{0}+O\left(\left(s-\hat{s}_{0}\right)^{2}\right)
$$

where the suffix 0 refers to the initial values. A similar result may be obtained for $g_{2}(r, s)$. Consequently, when $r$ and $s$ differ only slightly from constant values $\hat{r}_{0}$ and $\hat{s}_{0}$ we may use as our estimates of $t_{\mathrm{inf}}$ and $t_{\mathrm{sup}}$ the alternative expressions: 
$t_{\mathrm{inf}}$ is the least positive number of the two quantities

$$
\begin{aligned}
& \frac{-1}{\max _{r, 0}\left[\left(\frac{\partial \lambda^{(1)}}{\partial r}\right) \exp \left\{\left(\frac{\hat{s}_{0}-s}{\lambda_{0}^{(1)}-\lambda_{0}^{(2)}}\right)\left(\frac{\partial \lambda^{(1)}}{\partial s}\right)_{0}\right\}\right] \cdot \max \left(\frac{\partial r}{\partial x}\right)_{t=0}}, \\
& \frac{-1}{\max _{r, 0}\left[\left(\frac{\partial \lambda^{(2)}}{\partial s}\right) \exp \left\{\left(\frac{\hat{r}_{0}-r}{\lambda_{0}^{(2)}-\lambda_{0}^{(1)}}\right)\left(\frac{\partial \lambda^{(2)}}{\partial r}\right)_{0}\right\}\right] \cdot \max \left(\frac{\partial s}{\partial x}\right)_{t=0}}
\end{aligned}
$$

and

$t_{\text {sup }}$ is the least positive number of the two quantities

$$
\begin{aligned}
& \min _{r, 0}\left[\left(\frac{\partial \lambda^{(1)}}{\partial r}\right) \exp \left\{\left(\frac{\hat{s}_{0}-s}{\lambda_{0}^{(1)}-\lambda_{0}^{(2)}}\right)\left(\frac{\partial \lambda^{(1)}}{\partial s}\right)_{0}\right\}\right] \cdot \max \left(\frac{\partial r}{\partial x}\right)_{t=0}, \\
& \frac{-1}{\min _{r, s}\left[\left(\frac{\partial \lambda^{(2)}}{\partial s}\right) \exp \left\{\left(\frac{\hat{r}_{0}-r}{\lambda_{0}^{(2)}-\lambda_{0}^{(1)}}\right)\left(\frac{\partial \lambda^{(2)}}{\partial r}\right)_{0}\right\}\right] \cdot \max \left(\frac{\partial s}{\partial x}\right)_{t=0}} .
\end{aligned}
$$

It is at once obvious that the solution will only break down due to the $C^{(1)}$ characteristics when $\max (\partial r / \partial x)_{t=0}>0$ and, similarly, it will only break down due to the $C^{(2)}$ characteristics when $\max (\partial s / \partial x)_{t=0}>0$.

A much simplified version of these results was obtained by Lax [14] using different comparison theorems in which he assumed that $r=\hat{r}_{0}, s=\hat{s}_{0}$ in order to study the existence of solutions of a certain nonlinear string equation.

5. Gas motion in a closed tube. Ludford [9] considered the mixed initial and boundary value problem that is involved in describing the one-dimensional motion of a polytropic gas enclosed between the two fixed walls $x=0$ and $x=l$ when the initial density and velocity variation are specified. We shall now apply the results of the previous Section to this problem and, as in Ludford's paper, convert the problem to a pure initial value problem by suitable periodic extension of the solution outside the fundamental interval $[0, l]$.

The equations of conservation of mass and momentum may be written in the matrix form

$$
U_{t}+A U_{x}=0
$$

in which

$$
U=\left[\begin{array}{l}
\rho \\
u
\end{array}\right] \text { and } \quad A=\left[\begin{array}{cc}
u & \rho \\
a^{2} / \rho & u
\end{array}\right] .
$$

Here $\rho$ is the density, $u$ is the gas velocity and $a^{2}=\partial p / \partial \rho$ is the square of the sound speed in the gas which, if polytropic, has a pressure $p$ that is determined 
by the relation

$$
p=A \rho^{\gamma}
$$

with $A$ and $\gamma$ positive constants.

The characteristic roots $\lambda^{(i)}$ and the corresponding left characteristic vectors $l^{(i)}$ of the matrix $A$ are easily seen to be

$$
\lambda^{(1)}=u+a, \quad \lambda^{(2)}=u-a
$$

and

$$
l^{(1)}=\left[\frac{a}{\rho}, 1\right], \quad l^{(2)}=\left[\frac{a}{\rho},-1\right],
$$

respectively. Using results (5.4) in equations (1.10) and (1.11) and recognising that the integrating factors $q_{1}=q_{2}=1$, we then find that along the $C^{(1)}$ characteristics

$$
C^{(1)}: \frac{d x}{d t}=u+a
$$

we have the Riemann invariant relation

$$
u+\int \frac{a}{\rho} d \rho=-r
$$

while along the $C^{(2)}$ characteristics

$$
C^{(2)}: \frac{d x}{d t}=u-a
$$

we have the Riemann invariant relation

$$
u-\int \frac{a}{\rho} d \rho=-s,
$$

where the minus signs are introduced to make $\partial \lambda^{(1)} / \partial r$ and $\partial \lambda^{(2)} / \partial s$ negative as required.

To utilize the estimates of the critical time provided in expressions (4.14) to (4.17) we need to determine $\left(\partial \lambda^{(1)} / \partial r\right),\left(\partial \lambda^{(1)} / \partial s\right),\left(\partial \lambda^{(2)} / \partial r\right)$ and $\left(\partial \lambda^{(2)} / \partial s\right)$. We shall only discuss the determination of the first two of these expressions as the determination of the others is similar apart from the notation.

As $\lambda^{(1)}=u+a$, and so

$$
\frac{\partial \lambda^{(1)}}{\partial r}=\frac{\partial u}{\partial r}+\left(\frac{d a}{d \rho}\right) \frac{\partial \rho}{\partial r}
$$

it follows that we need to determine $\partial u / \partial r$ and $\partial \rho / \partial r$. Now for a gas obeying the polytropic gas law (5.2) we have $a^{2}=A \gamma \rho^{(\gamma-1)}$, and so $d a / d \rho=(a / 2 \rho)(\gamma-1)$. Using the first of these results then shows that 


$$
\int \frac{a}{\rho} d \rho=\left(\frac{2 a}{\gamma-1}\right) .
$$

Adding and subtracting equations (5.6) and (5.7) thus gives rise to the two equations

$$
\begin{aligned}
& r+s=-2 u, \\
& r-s=\frac{-4 a}{(\gamma-1)} .
\end{aligned}
$$

Differentiating $(r+s)$ with respect to $r$ immediately gives

$$
\frac{\partial u}{\partial r}=-\frac{1}{2},
$$

while differentiating $(r-s)$ with respect to $r$ gives

$$
1=\left(\frac{-4}{\gamma-1}\right)\left(\frac{d a}{d \rho}\right)\left(\frac{\partial \rho}{\partial r}\right) .
$$

So, combining equations (5.9), (5.13) and (5.14), we find that

$$
\frac{\partial \lambda^{(1)}}{\partial r}=-\left(\frac{\gamma+1}{4}\right)
$$

which is, in fact, independent of $r$ and $s$.

Similar reasoning also shows that

$$
\frac{\partial \lambda^{(1)}}{\partial s}=\frac{\gamma-3}{4},
$$

which is also independent of $r$ and $s$.

To apply the estimates stated in (4.14) to (4.17) we must first convert the combined initial and boundary value problem for the gas motion to a pure initial value problem. The specification of the initial values of $u$ and $\rho$ on $[0, l]$ determines the initial values of $r_{0}(x)=r(x, 0)$ and $s_{0}(x)=s(x, 0)$ on this interval. The boundary conditions that are to be imposed are $u(0, t)=u(l, t)=0$ for all $t$. So, by virtue of (5.11), we may write

$$
r_{0}(0)+s_{0}(0)=r_{0}(l)+s_{0}(l)=0 .
$$

Accordingly then we extend $r_{0}(x)+s_{0}(x)$ to the interval $[-l, l]$, and thence to the entire initial line, by defining it to be an even function in $[-l, l]$ that is periodic with period $2 l$. The function $r_{0}(x)-s_{0}(x)$ is similarly extended as an odd function in $[-l, l]$ which is periodic with period $2 l$. The boundary conditions on $u$ may now be disregarded since with this extension of $r_{0}(x)$ and $s_{0}(x)$ the problem becomes a pure initial value problem.

Considering the pure initial value problem just defined and using the estimates (4.14) and (4.15) we see that when $\max (\partial r / \partial x)_{t=0}$ and $\max (\partial s / \partial x)_{t-0}$ are positive, $t_{\text {in }}$ is the lesser of the two numbers 


$$
\begin{aligned}
& \frac{4}{(\gamma+1) \max _{\cdot}\left[\exp \left\{\frac{(\gamma-3)\left(\hat{s}_{0}-s\right)}{8 a_{0}}\right\}\right] \cdot \max \left(\frac{\partial r_{0}(x)}{\partial x}\right)}, \\
& \frac{4}{(\gamma+1) \max _{r}\left[\exp \left\{\frac{(3-\gamma)\left(\hat{r}_{0}-r\right)}{8 a_{0}}\right\}\right] \cdot \max \left(\frac{\partial s_{0}(x)}{\partial x}\right)}
\end{aligned}
$$

Similarly, from estimates (4.16) and (4.17) we find that $t_{\mathrm{sup}}$ is the lesser of the two numbers

$$
\begin{aligned}
& \frac{4}{(\gamma+1) \min _{\bullet}\left[\exp \left\{\frac{(\gamma-3)\left(\hat{s}_{0}-s\right)}{8 a_{0}}\right\}\right] \cdot \max \left(\frac{\partial r_{0}(x)}{\partial x}\right)}, \\
& \frac{4}{(\gamma+1) \min _{r}\left[\exp \left\{\frac{(3-\gamma)\left(\hat{r}_{0}-r\right)}{8 a_{0}}\right\}\right] \cdot \max \left(\frac{\partial s_{0}(x)}{\partial x}\right)}
\end{aligned}
$$

If $r$ and $s$ in these expressions are replaced by their constant values $\hat{r}_{0}$ and $\hat{s}_{0}$, as was done by Ludford [9] so that $r=\hat{r}_{0}, s=\hat{s}_{0}$, the numbers $t_{\text {inf }}$ and $t_{\text {sup }}$ then coincide. Then, denoting by $\beta$ the greater of the two quantities $\max \left[\partial r_{0}(x) / \partial x\right]$ and $\max \left[\partial s_{0}(x) / \partial x\right]$, we obtain the estimate

$$
t_{c}=\frac{4}{(\gamma+1) \beta}
$$

This is exactly the simple result obtained by Ludford when allowance is made for the fact that his definitions of the Riemann invariants $r$ and $s$ differ by a numerical factor two from those given in equations (5.6) and (5.7).

6. Waves in shallow water. When waves occur in shallow water of constant depth the equations governing the fluid motion may be written in the form [11]

$$
U_{t}+A U_{x}=0
$$

where

$$
U=\left[\begin{array}{l}
u \\
c
\end{array}\right], \quad A=\left[\begin{array}{cc}
u & 2 c \\
\frac{1}{2} c & u
\end{array}\right] .
$$

Here $u$ denotes the $x$-component of the fluid velocity and $c$ the local wave propagation speed $(g y(x))^{1 / 2}$ at a point with coordinate $x$, where $y(x)$ is the depth of the water and $g$ is the acceleration due to gravity.

The characteristic roots of $A$ are

$$
\lambda^{(1)}=u+c, \quad \lambda^{(2)}=u-c
$$

and the left characteristic vectors are 


$$
l^{(1)}=[1,2], \quad l^{(2)}=[1,-2] .
$$

The Riemann invariants then take the form

$$
u+2 c=-r
$$

along the $C^{(1)}$ characteristics

$$
C^{(1)}: \frac{d x}{d t}=u+c
$$

and

$$
u-2 c=-s
$$

along the $C^{(2)}$ characteristics

$$
C^{(2)}: \frac{d x}{d t}=u-c,
$$

the minus signs being introduced to make $\partial \lambda^{(1)} / \partial r$ and $\partial \lambda^{(2)} / \partial s$ negative.

This problem is a pure initial value problem but, as in Section 5, we need to determine $\left(\partial \lambda^{(1)} / \partial r\right),\left(\partial \lambda^{(1)} / \partial s\right),\left(\partial \lambda^{(2)} / \partial r\right)$ and $\left(\partial \lambda^{(2)} / \partial s\right)$ in order to utilize the estimates contained in Section 4.

From (6.3) we see that

$$
\frac{\partial \lambda^{(1)}}{\partial r}=\frac{\partial u}{\partial r}+\frac{\partial c}{\partial r},
$$

but adding and subtracting equations (6.5) and (6.7) and differentiating with respect to $r$ shows that $\partial c / \partial r=-\frac{1}{4}, \partial c / \partial s=\frac{1}{4}, \partial u / \partial r=-\frac{1}{2}$ and $\partial u / \partial s=-\frac{1}{2}$ so that

$$
\frac{\partial \lambda^{(1)}}{\partial r}=-\frac{3}{4}, \quad \frac{\partial \lambda^{(1)}}{\partial s}=-\frac{1}{4}, \quad \frac{\partial \lambda^{(2)}}{\partial r}=-\frac{1}{4}, \quad \frac{\partial \lambda^{(2)}}{\partial s}=-\frac{3}{4},
$$

which are all independent of $r$ and $s$.

Then from estimates (4.14) to (4.17) we see that provided $\max \left[\partial r_{0}(x) / \partial x\right]$ and $\max \left[\partial s_{0}(x) / \partial x\right]$ are positive:

$t_{\mathrm{in} \boldsymbol{t}}$ is the lesser of the two numbers

$$
\frac{4}{3 \max \left[\exp \left(\frac{s-\hat{s}_{0}}{8 c_{0}}\right)\right] \cdot \max \left(\frac{\partial r_{0}(x)}{\partial x}\right)}, \frac{4}{3 \max \left[\exp \left(\frac{\hat{r}_{0}-r}{8 c_{0}}\right)\right] \cdot \max \left(\frac{\partial s_{0}(x)}{\partial x}\right)}
$$

and $t_{\text {sup }}$ is the lesser of the two numbers

$$
\frac{4}{3 \min \left[\exp \left(\frac{s-\hat{s}_{0}}{8 c_{0}}\right)\right] \cdot \max \left(\frac{\partial r_{0}(x)}{\partial x}\right)}, \frac{4}{3 \min _{r}\left[\exp \left(\frac{\hat{r}_{0}-r}{8 c_{0}}\right)\right] \cdot \max \left(\frac{\partial s_{0}(x)}{\partial x}\right)} .
$$

Again denoting by $\beta$ the larger of the two values $\max \left[\partial r_{0}(x) / \partial x\right]$ and 
$\max \left[\partial s_{0}(x) / \partial x\right]$ we see that in the limit as $r$ and $s$ tend towards $\hat{r}_{0}$ and $\hat{s}_{0}$ the estimates $t_{\mathrm{inf}}$ and $t_{\mathrm{sup}}$ coincide to give the estimate

$$
t_{c}=\frac{4}{3 \beta}
$$

This result can now be applied to the problem in which a wave advances into water which is at rest above a horizontal sea bed, so that a Lipschitz discontinuity occurs across the wavefront. As would be expected, the vanishing of $\partial r / \partial x$ and $\partial s / \partial x$ in the region ahead of the wave implies, via the estimates (6.11) and (6.12), that only the disturbed region will influence the occurrence of a discontinuity in the solution. To examine the breaking of waves at the wavefront we only need to examine the forward facing disturbance that propagates along the $C^{(1)}$ characteristic $C_{0}^{(1)}$, say, that is followed by the wavefront. Assuming that at time $t=0$ the wavefront is at $x=0$, our estimate of time $t_{c}$ will be given by

$$
t_{c}=\frac{4}{3\left(\frac{\partial r_{0}(x)}{\partial x}\right)_{x=0}} .
$$

From the definition of the Riemann invariant $r$ in equation (6.5) we see that

$$
\frac{\partial r}{\partial x}=-\left(\frac{\partial u}{\partial x}+2 \frac{\partial c}{\partial x}\right)
$$

Now by its method of definition, the Riemann invariant $s$ in equation (6.7) is constant along each $C^{(2)}$ characteristic, the value of the constant being equal to the value that is assumed by $s$ in some known state. Thus, as all the $C^{(2)}$ characteristics enter the same constant state, it follows immediately that in this case $s$ must be an absolute constant independent of the $C^{(2)}$ characteristics so that equation (6.7) simplifies to

$$
u-2 c=u_{0}-2 c_{0}=\text { constant, }
$$

the suffix 0 denoting a value appropriate to the constant state.

Differentiating this with respect to $x$ and substituting the result in (6.15) then shows that

$$
\frac{\partial r}{\partial x}=-4 \frac{\partial c}{\partial x}
$$

so that estimate $(6.14)$ becomes

$$
t_{c}=\frac{-1}{3\left[\frac{\partial c}{\partial x}\right]_{\substack{x=0 \\ t=0}}}
$$

When, as was considered by Jeffrey [12] and Stoker [15], a sinusoidal wave 
disturbance of amplitude $A \sin \omega t$ is incident at the origin then $c_{0}(0, t)$, the wave propagation speed at the origin, is given by

$$
c_{0}(0, t)=(g(h+A \sin \omega t))^{1 / 2},
$$

where $h$ is the constant depth of the undisturbed water.

Then, since along $C^{(1)}$ characteristics

$$
\frac{\partial c}{\partial x} d x+\frac{\partial c}{\partial t} d t=0 \quad \text { such that } \quad \frac{d x}{d t}=u+c
$$

we must have

$$
\frac{\partial c}{\partial x}=-\left(\frac{1}{u+c}\right) \frac{\partial c}{\partial t}
$$

Setting $\bar{c}_{0}=(g h)^{1 / 2}$, the initial speed of propagation of the wavefront, and using equation (6.19) to determine $\partial c / \partial t$ at the time the wavefront passes $x=0$ we find that

$$
\left(\frac{\partial c}{\partial x}\right)_{\substack{x=0 \\ t=0}}=\frac{-g A \omega}{2 \bar{c}_{0}\left(\bar{c}_{0}+u_{0}\right)} .
$$

When used in equation (6.18) this gives the result

$$
t_{c}=\frac{2 \bar{c}_{0}\left(\bar{c}_{0}+u_{0}\right)}{3 g A \omega},
$$

which was obtained by two independent methods in references [12, 15]. The fact that $t_{c}$ will only be positive, and so actual breaking of waves will occur on the wavefront, when $A>0$, confirms Stoker's result [13, Sections 10 and 11] that a depression wave of this type in which $A<0$ will not break on the wavefront.

7. Nonlinear electromagnetic transmission line. In this final section we shall consider the development of electromagnetic shock waves in a simple distributed parameter non-dissipative transmission line. When the nonlinearity is confined to a voltage dependent capacitance the equations governing propagation become

$$
U_{t}+A U_{x}=0
$$

with

$$
U=\left[\begin{array}{l}
i \\
v
\end{array}\right], \quad A=\left[\begin{array}{cc}
0 & 1 / L \\
1 / C & 0
\end{array}\right] .
$$

Here $i$ denotes the current, $v$ the voltage, $L$ the inductance and $C(v)$ the voltage dependent capacitance.

The characteristic roots of $A$ are 


$$
\lambda^{(1)}=\frac{1}{(L C)^{1 / 2}}, \quad \lambda^{(2)}=\frac{-1}{(L C)^{1 / 2}}
$$

and they determine the characteristic curves

$$
C^{(1)}: \frac{d x}{d t}=\frac{1}{(L C)^{1 / 2}} \quad \text { and } \quad C^{(2)}: \frac{d x}{d t}=\frac{-1}{(L C)^{1 / 2}} .
$$

The corresponding left characteristic vectors $l^{(i)}$ are

$$
l^{(1)}=\left[1,\left(\frac{C}{L}\right)^{1 / 2}\right], \quad l^{(2)}=\left[1,-\left(\frac{C}{L}\right)^{1 / 2}\right]
$$

which determine, through equations (1.10) and (1.11), the Riemann invariants

$$
i+\int\left(\frac{C}{L}\right)^{1 / 2} d v=r \quad \text { along } C^{(1)} \text { characteristics, }
$$

and

$$
i-\int\left(\frac{C}{L}\right)^{1 / 2} d v=s \quad \text { along } C^{(2)} \text { characteristics. }
$$

Subtracting equation (7.7) from (7.6) gives

$$
2 \int\left(\frac{C}{L}\right)^{1 / 2} d v=r-s
$$

and differentiating with respect $r$ then shows that

$$
\frac{\partial v}{\partial r}=\frac{1}{2}\left(\frac{L}{C}\right)^{1 / 2} .
$$

As $\lambda^{(1)}$ is only a function of $v$ we may write

$$
\frac{\partial \lambda^{(1)}}{\partial r}=\left(\frac{d \lambda^{(1)}}{d v}\right)\left(\frac{\partial v}{\partial r}\right)
$$

so that using (7.3) and (7.8) we obtain

$$
\frac{\partial \lambda^{(1)}}{\partial r}=-\frac{1}{4 C^{2}}\left(\frac{\partial C}{\partial v}\right)
$$

which is negative, as required, provided $(\partial C / \partial v)$ is positive. (Otherwise the sign of $r$ must be changed.)

For simplicity of argument we shall assume that $r$ and $s$ differ only infinitesimally from $\hat{r}_{0}$ and $\hat{s}_{0}$, so that $t_{\text {int }}$ and $t_{\text {sup }}$ coincide with $t_{c}$ which is the smaller of the two numbers

$$
\frac{4 C_{0}^{2}}{\left(\frac{\partial C}{\partial v}\right)_{\substack{r=\hat{f}_{0} \\ j=\hat{o}_{0}}} \max \left(\frac{\partial r_{0}(x)}{\partial x}\right)}, \frac{4 C_{0}^{2}}{\left(\frac{\partial C}{\partial v}\right)_{\substack{r=\hat{f}_{0} \\ j=\hat{s}_{0}}} \max \left(\frac{\partial s_{0}(x)}{\partial x}\right)},
$$


where $C_{0}$ denotes the value assumed by $C$ when $r=\hat{r}_{0}$ and $s=\hat{s}_{0}$. It is immediately apparent that a discontinuity will occur (i.e., an electromagnetic shock wave will form) only when the signs of the denominators in (7.10) are positive.

In the case of Lipschitz continuous initial data in which a wave advances into a constant state the resulting wave motion will in fact be described, as in Section 6, by simple waves [7]. As before $s$ will then be an absolute constant so that differentiation of (7.7) with respect to $x$ will give

$$
\frac{\partial i}{\partial x}=\left(\frac{C}{L}\right)^{1 / 2} \frac{\partial v}{\partial x} .
$$

So, differentiating $r$ in equation (7.6) with respect to $x$ and using (7.11) we find that

$$
\frac{\partial r}{\partial x}=2 \frac{\partial i}{\partial x} .
$$

The formation of a discontinuity on the $C^{(1)}$ characteristic that forms the wavefront will thus occur at the time

$$
t_{c}=\frac{2 C_{0}^{2}}{\left(\frac{\partial i_{0}(x)}{\partial x}\right)\left(\frac{\partial C}{\partial v}\right)_{\substack{r=\hat{f}_{0} \\ s=\hat{o}_{0}}} .}
$$

\section{REFERENCES}

[1] Courant, R. \& Friedrichs, K. O., Supersonic Flow and Shock Waves, Interscience, 1948, Sections 48 and 49.

[2] Friedrichs, K. O., Nonlinear hyperbolic differential equations for functions of two independent variables, Am. J. Math., 70 (1948) 555-589.

[3] LAx, P. D., The initial value problem for non-linear hyperbolic equations in two independent variables, Ann. Math. Studies (Princeton), 33 (1954) 211-229.

[4] Thomas, T. Y., The growth and decay of sonic discontinuities in ideal gases, J. Math. and Mech., 6 (1957) 455-469.

[5] Jefrrey, A., The development of jump discontinuities in nonlinear hyperbolic systems of equations in two independent variables, Arch. Rat. Mech. Anal., 14 (1963) 27-37.

[6] JEFFrex, A., The development of singularities of solutions of nonlinear hyperbolic equations of order greater than unity, J. Math. and Mech., 15 (1966) 585-598.

[7] Jefrrey, A. \& Taniutr, T., Nonlinear Wave Propagation, Academic Press, 1964, Chapter 2.

[8] Riemann, B., Ưber die Fortpflanzung ebener Luftwellen von enlicher Schwingungsweite, Abh. Ges. Wiss. Göttingen, 8 (1860) 43.

[9] LUDFord, G. S. S., On an extension of Riemann's method of integration, with applications to one-dimensional gas dynamics, Proc. Camb. Phil. Soc., 48 (1952) 499-510.

[10] Stocker, P. \& Meyer, R. E., A note on the correspondence between the $(x, t)$-plane and the characteristic plane in a problem of interaction of plane waves of finite amplitude, Proc. Camb. Phil. Soc., 47 (1951) 518-527.

[11] STоKer, J. J., Water Waves, Interscience, 1957, Section 10.

[12] Jefrrey, A., The breaking of waves on a sloping beach., Zeit. Ang. Math. u. Phys., 15 (1964) $97-106$. 
[13] Codpington, E. A. \& Levinson, N., Theory of Ordinary Differential Equations, McGrawHill, 1955.

[14] LAX, P. D., Development of singularities of solutions of nonlinear hyperbolic partial differential equations, J. Math. Phys., 5 (1964) 611-613.

[15] Stoker, J. J., The formation of breakers and bores, Comm. Pure Appl. Math., 1 (1948) 1-87 (Section 11).

University of Newcastle upon Tyne, England

Date Communicated: February 7, 1967 